\title{
Disección endoscópica submucosa para el tratamiento del cáncer esofágico
}

\author{
Ricardo Mejía M. ${ }^{1}$, Josefina Sáez B. ${ }^{2}$, Daniel Moreno ${ }^{2}$, Enrique Norero M. ${ }^{1,3}$, \\ Marco Ceroni V. ${ }^{3}$, Pamela Briones ${ }^{3}$, Ignacio Obaid C. ${ }^{3}$, Cristian Martínez B. ${ }^{3}$, \\ Rodrigo Muñoz C..$^{1,3}$, Constanza Godoy S. ${ }^{3}$, Alfonso Díaz F. ${ }^{3}$ y Allan Sharp P. ${ }^{1}$
}

\section{Endoscopic submucosal dissection for the treatment of esophageal cancer}

Introduction: Esophageal cancer is the sixth leading cause of death by cancer worldwide. Endoscopic submucosal dissection (ESD) is a technique that allows en bloc resection of early lesions of the digestive tract. It has curative potential in selected patients and potential benefits over esophagectomy for the treatment of esophageal cancer (EC). Aim: To report the results of ESD for EC in a high-volume center in Chile. Materials and Method: Retrospective descriptive study of patients who underwent ESD for EC at our center. Results: A total of 10 patients were treated with ESD for EC between 2010 and 2020. Eighty percent were male patients, the average age was 72 years (63-84). En bloc resection was achieved in all cases and there were no complications or mortality. All of the dissections were classified as R0 and 90\% met curative standards. The mean follow-up was 38 months (3.5-123). Overall survival was 90\%. Cancer-specific survival was 100\% and recurrence-free survival was 100\%. Discussion: The rate of morbidity and mortality of esophagectomy is high. ESD would be a safer technique, which allows R0 resections and eventually curation in selected patients with EC limited to the mucosa or submucosa. Conclusion: This is the first report of ESD for the treatment of EC in our country, it shows excellent oncologic results and safety of the procedure, comparable to those reported in the larger series published to date.

Key words: esophagus; esophageal neoplasms; endoscopy; endoscopic submucosal dissection; therapeutics.

\section{Resumen}

Introducción: El cáncer de esófago (CE) constituye la sexta causa de muerte por cáncer en el mundo. La disección endoscópica submucosa (DES) es una técnica que permite la resección en bloque de lesiones del tubo digestivo. Tiene rol curativo en pacientes seleccionados y potenciales ventajas sobre la esofagectomía. Objetivo: Describir los resultados perioperatorios y oncológicos de la DES como tratamiento del CE en nuestro centro. Materiales y Método: Estudio retrospectivo de pacientes sometidos a DES por CE entre los años 2010-2020. Resultados: Diez pacientes fueron tratados con DES por CE entre los años 2010 y 2020. El 80\% eran hombres y la edad promedio fue de 72 años (63-84). La resección en bloque fue lograda en todos los casos y no se presentó morbimortalidad perioperatoria. Todas las disecciones fueron R0 y el 90\% cumplió con estándares de curación. El seguimiento promedio fue de 38 meses (3,5-123). La sobrevida global fue de 90\%. La sobrevida específica por cáncer y libre de recurrencia fue de $100 \%$. Discusión: La morbimortalidad asociada a la esofagectomía es alta. La DES sería una alternativa más segura, que permite lograr un R0 y eventualmente la curación en pacientes seleccionados con CE limitado a la mucosa o submucosa. Conclusión: La presente constituye la primera serie reportada de pacientes con cáncer esofágico sometidos a DES en nuestro país. Muestra excelentes resultados oncológicos y seguridad del procedimiento, comparables a las grandes series descritas en la literatura internacional.

Palabras clave: esófago; cáncer de esófago; endoscopia; disección endoscópica submucosa; tratamiento.
'Departamento de Cirugía Digestiva, División de Cirugía, Facultad de Medicina, Pontificia Universidad Católica de Chile.

2Escuela de Medicina, Facultad de Medicina, Pontificia

Universidad Católica de Chile. ${ }^{3}$ Cirugía Esófago-Gástrica, Unidad Docente Asistencial, Complejo Asistencial Doctor Sótero del Río.

Recibido 2020-06-2 y aceptado 2020-06-23

Correspondencia a: Dr. Ricardo Mejía M. rjmejiam@gmail.com 


\section{Introducción}

El cáncer de esófago constituye la sexta causa de muerte por cáncer en el mundo'. En Chile es el duodécimo cáncer más frecuente para la población masculina y el décimo cuarto para la población femenina ${ }^{2}$. Históricamente el tipo histológico predominante fue el carcinoma escamoso. Sin embargo, en los últimos 20 años ha aumentado la proporción de adenocarcinomas, alcanzando actualmente al $60 \%$ de los cánceres de esófago en occidente ${ }^{3}$.

La sobrevida global a 5 años es alrededor de un $20 \%$, lo que principalmente se explica porque hasta un $60 \%$ de los pacientes se presenta en estadios avanzados al momento del diagnóstico ${ }^{4}$.

La esofagectomía con disección ganglionar constituye el estándar de tratamiento en pacientes con enfermedad localizada ${ }^{5}$. Es una de las cirugías electivas con mayor mortalidad perioperatoria, alcanzando un $13 \%$ en centros con bajo volumen de casos y alrededor de $5 \%$ en aquellos de alto volumen ${ }^{5-8}$. La morbilidad reportada varía entre un 50\%-75\%, siendo las principales complicaciones las respiratorias, que incluso superan el $30 \%$ en cirugía abierta ${ }^{6-9}$.

Dada la alta morbimortalidad asociada a la esofagectomía, desde 1990 se han dirigido esfuerzos a desarrollar alternativas endoscópicas para el diagnóstico y tratamiento de lesiones esofágicas ${ }^{10}$. La mucosectomía esofágica (EMR, por sus siglas del inglés) permite la resección en bloque de lesiones pequeñas, pero su uso en lesiones de mayor tamaño se asocia a resecciones en varios fragmentos ${ }^{11}$. Lo anterior se traduce en un riesgo de recidiva cercano al $25 \%{ }^{12} \mathrm{y}$, además, a dificultades en el análisis patológico de la pieza quirúrgica. Esto impide una adecuada etapificación y/o determinar la curación aun cuando la lesión haya sido removida completamente ${ }^{11}$.

La disección endoscópica submucosa (DES o ESD por su sigla en inglés) es una técnica descrita en los años 90 en Japón que permite la resección en bloque, independiente del tamaño, de lesiones incipientes del tubo digestivo ${ }^{13}$. Tendría potencial curativo en pacientes seleccionados, una baja morbimortalidad y menor impacto sobre la calidad de vida, lo cual supondría claras ventajas por sobre la esofagectomía ${ }^{11}$.

Existe una sólida evidencia en términos de los resultados oncológicos de la DES para el tratamiento del cáncer gástrico incipiente (CGI), que la posicionan como una técnica segura y con resultados comparables a la cirugía radical ${ }^{14,15}$. En cáncer de esófago la experiencia reportada es menor y la diseminación linfática ocurre en etapas más tempranas de la enfermedad, lo que obliga a ser aún más estrictos con la indicación para considerar a una resección como curativa ${ }^{16}$.

El objetivo del presente trabajo es describir los resultados perioperatorios y oncológicos de la DES como tratamiento del cáncer esofágico en nuestro centro.

\section{Materiales y Método}

Estudio retrospectivo descriptivo que incluyó a pacientes sometidos a DES con intención curativa por cáncer de esófago en el Complejo Asistencial Dr. Sótero del Río y Red de Salud UC Christus en el período comprendido entre septiembre de 2010 y febrero de 2020.

La indicación de resección endoscópica fue evaluada en un comité multidisciplinario luego de una discusión individualizada y contando con una adecuada etapificación clínica. Todas las resecciones fueron realizadas por cirujanos dedicados a endoscopía terapéutica y con experiencia en DES del tracto digestivo.

Se registraron datos epidemiológicos y perioperatorios, obtenidos desde el registro institucional.

La evaluación preoperatoria incluyó una evaluación clínica, hemograma, pruebas de función hepática y renal y electrocardiograma. El diagnóstico fue realizado con endoscopía digestiva alta con biopsia de la lesión y la etapificación incluyó una tomografía de tórax, abdomen y pelvis con contraste endovenoso y tomografía por emisión de positrones (PET) desde cuando este examen estuvo disponible.

\section{Técnica quirúrgica}

Todos los pacientes fueron sometidos a la DSE en pabellón, con anestesia general y relajo muscular, en decúbito lateral izquierdo. Se utilizó un endoscopio diagnóstico Olympus ${ }^{\circledR}$ serie 180 o 190 con un espaciador distal. La insuflación fue realizada con $\mathrm{CO} 2$.

Luego de identificar la lesión y sus márgenes mediante endoscopía de luz blanca y cromoendoscopía (índigo carmín o lugol), se procedió a la marcación de la periferia de ésta con electrocoagulación. Se realizó la elevación del espacio submucoso con una solución constituida por voluven ${ }^{\circledR}$, índigo carmín y adrenalina. La mucotomía y posterior disección submucosa se llevaron a cabo con cuchillos endoscópicos procurando la resección en bloque de la pieza (Figura 1). Al terminar el procedimiento se realizó hemostasia del lecho con pinzas de coagulación y se verificó la indemnidad de la pared esofágica. 


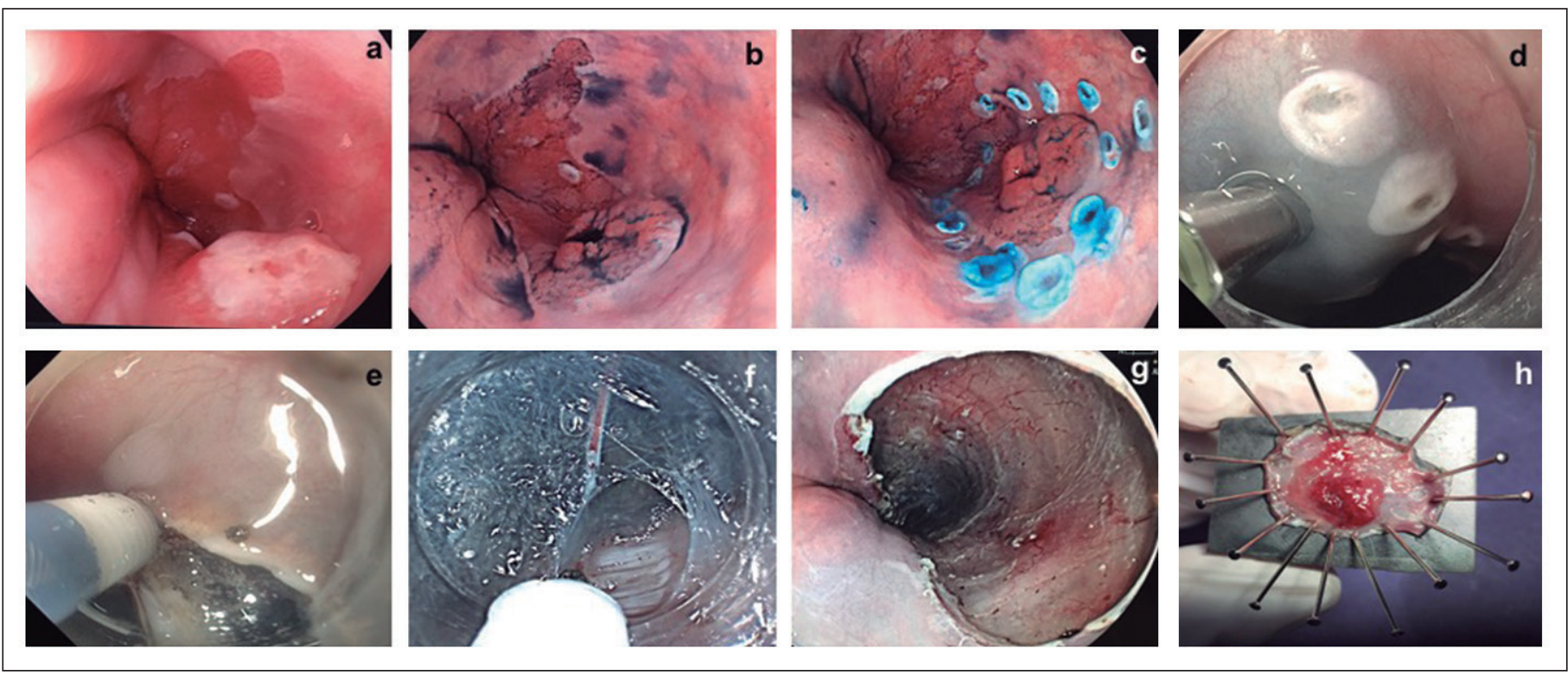

Figura 1. Técnica de DSE. a: Endoscopía de luz blanca; b: Tinción con índigo carmín y c: Marcación de la lesión; d: Elevación de la submucosa; e: Mucotomía; f: Disección submucosa; g: Lecho de resección; h: Pieza extendida.

La pieza fue extendida, fijada en sus extremos y enviada en formalina para su estudio anatomopatológico definitivo (Figura 1). Se evaluó dirigidamente el tipo histológico, tamaño tumoral, grado de diferenciación, presencia de permeaciones linfovasculares y los bordes laterales y profundo.

Los pacientes fueron trasladados a una unidad quirúrgica de baja complejidad y mantenidos las primeras $24 \mathrm{~h}$ en régimen cero, con hidratación endovenosa, inhibidores de bomba de protones (IBP), antieméticos y analgesia multimodal. Se evitó el uso de anticoagulantes y se favoreció la deambulación precoz.

El día posterior a la cirugía se inició una dieta líquida y la analgesia e IBP administrados por vía oral. El alta fue indicada a las $48 \mathrm{~h}$ con régimen papilla que fue mantenido hasta el control ambulatorio.

\section{Seguimiento}

El seguimiento fue realizado mediante control clínico, endoscópico y biopsias rutinarias a los 3, 6 y 12 meses. Posteriormente, los pacientes se mantuvieron en control endoscópico anual.

\section{Análisis de los datos}

Fueron analizadas variables demográficas, características clínicas, del diagnóstico endoscópico e histológico preoperatorio, datos del procedimiento y morbilidad asociada, resultado histológico definitivo y seguimiento. Se evaluó la sobrevida global y libre de enfermedad.

Las variables categóricas se expresan en valores numéricos y porcentajes, las variables continuas en medianas y rangos. El análisis se realizó con SPSS v24, Inc, Chicago, IL.

El presente trabajo fue aprobado por el comité de ética local.

\section{Resultados}

Un total de 10 pacientes fueron sometidos a una DES por cáncer de esófago entre el año 2010 y 2020. El $80 \%$ corresponde a pacientes de género masculino y la edad promedio fue de 72 años (6384). Un $90 \%$ correspondían a categoría ASA II y $10 \%$ a ASA III (Tabla 1 ).

La histología preoperatoria incluyó 4 adenocarcinomas, 4 carcinomas escamosos y 2 lesiones con displasia de alto grado (DAG) (Tabla 2).

La resección en bloque fue lograda en todos los casos. No se presentó ninguna morbilidad ni mortalidad perioperatoria. Un paciente evolucionó con una estenosis esofágica diagnosticada a las seis semanas luego del procedimiento, manejada satisfactoriamente con dilataciones endoscópicas. Cabe destacar que en dicho paciente la resección incluyó más del $75 \%$ de la circunferencia esofágica y fue tratado de manera profiláctica con un esquema de un mes de corticoides orales. 
Todos los pacientes fueron dados de alta al segundo día posoperatorio.

Todas las resecciones fueron catalogadas como R0. En un caso se informó compromiso submucoso profundo (1.600 micras) y el paciente fue sometido a una esofagectomía mínimamente invasiva como tratamiento definitivo. El estudio histológico final informó ausencia de tumor esofágico residual y linfonodos negativos $(0 / 39)$.

Las histologías posoperatorias incluyeron 4 adenocarcinomas bien diferenciados, 3 carcino-

\section{Tabla 1. Datos demográficos}

\begin{tabular}{|ll|}
\hline Género & Femenino 2 pacientes \\
& Masculino 8 pacientes \\
Edad & 72,3 años (rango 63-84 años) \\
& Cardiopatía coronaria $(1 / 10)$ \\
& Diabetes mellitus $(1 / 10)$ \\
& Enfermedad por reflujo gastroesofágico/ \\
& Esófago de Barrett $(4 / 10)$ \\
& Hipertensión arterial $(10 / 10)$ \\
& Linfoma No Hodgkin $(1 / 10)$ \\
& Tabaquismo activo $(3 / 10)$ \\
& Trastorno depresivo mayor $(1 / 10)$ \\
& ASA I $\quad-$ \\
ASA & ASA II 9 pacientes \\
& ASA III 1 paciente \\
\end{tabular}

mas escamosos bien diferenciados, 2 DAG y una displasia de bajo grado (Tabla 2). Solo el caso descrito previamente presentó compromiso submucoso, el resto de las DES fueron consideradas como curativas.

A la fecha no se han diagnosticado recurrencias. El promedio de seguimiento fue de 38 meses (3,5-123). La sobrevida global de la serie alcanzó un $90 \%$, solo registrándose un caso de mortalidad secundario a un accidente cerebrovascular. La sobrevida específica por cáncer esofágico fue de 100\% y la sobrevida libre de recurrencia fue un 100\%.

\section{Discusión}

El cáncer de esófago da cuenta de alrededor de 668 muertes al año en Chile ${ }^{2}$. La sobrevida global es baja y los malos resultados se explican en parte por el diagnóstico tardío, asociado a las malas condiciones generales y edad avanzada de los pacientes ${ }^{17}$. A lo anterior, se suma que en nuestro país anualmente se realizan alrededor de 63 esofagectomías $^{9}$ y un porcentaje menor de los centros logra acumular más de 10 casos anuales.

En el subgrupo de pacientes con cáncer esofágico confinado a la mucosa o submucosa el pronóstico oncológico es mejor, alcanzando un $70 \%$ de sobrevida a 5 años ${ }^{18}$. Es así como en esta etapa de la enfermedad el tratamiento endoscópico, de lograr un R0, presentaría claras ventajas por sobre la cirugía radical que con su sola ejecución conllevaría un riesgo de mortalidad del $10 \%$.

Tabla 2. Resultados anatomopatológicos

\begin{tabular}{|c|c|c|c|c|c|c|}
\hline \multirow{2}{*}{$\begin{array}{c}\text { Estudio } \\
\text { histológico }\end{array}$} & \multirow{2}{*}{ Preoperatorio } & \multicolumn{5}{|c|}{ Posoperatorio } \\
\hline & & Tipo & $\begin{array}{c}\text { Eje mayor } \\
(\mathrm{cm})\end{array}$ & $\begin{array}{l}\text { Profun- } \\
\text { didad }\end{array}$ & $\begin{array}{l}\text { Permeaciones } \\
\text { linfovasculares }\end{array}$ & Resección \\
\hline Paciente 1 & Displasia alto grado & Displasia alto grado & 0,5 & M1 & Ausentes & Curativa \\
\hline Paciente 2 & Carcinoma escamoso & Carcinoma escamoso & 0,4 & Sm1 & Ausentes & Curativa \\
\hline Paciente 3 & Carcinoma escamoso & Carcinoma escamoso & 1 & M1 & Ausentes & Curativa \\
\hline Paciente 4 & Adenocarcinoma bien diferenciado & Adenocarcinoma bien diferenciado & 1,6 & M3 & Ausentes & Curativa \\
\hline Paciente 5 & Displasia alto grado & Displasia alto grado & 0,5 & M1 & Ausentes & Curativa \\
\hline Paciente 6 & Displasia alto grado & Carcinoma escamoso & 0,4 & M1 & Ausentes & Curativa \\
\hline Paciente 7 & Displasia alto grado & Displasia bajo grado & 0,3 & M1 & Ausentes & Curativa \\
\hline Paciente 8 & Adenocarcinoma bien diferenciado & Adenocarcinoma bien diferenciado & 2,8 & $\mathrm{Sm} 3$ & Ausentes & No curativa \\
\hline Paciente 9 & Adenocarcinoma bien diferenciado & Adenocarcinoma bien diferenciado & 2,13 & M3 & Ausentes & Curativa \\
\hline Paciente 10 & Adenocarcinoma bien diferenciado & Adenocarcinoma bien diferenciado & 6 & M3 & Ausentes & Curativa \\
\hline
\end{tabular}


El tratamiento endoscópico del cáncer de esófago surge en Japón en los años 90 en donde se describen las primeras experiencias de EMR ${ }^{19-21}$. Posteriormente, y con el desarrollo de nuevo instrumental, en 2005 se reporta la primera DES para resección de una lesión esofágica ${ }^{22}$. Técnicamente la DES es un procedimiento más complejo que la EMR, más aún en el esófago en que se trabaja en un espacio reducido que dificulta la orientación espacial.

En occidente y principalmente en Norteamérica, se ha defendido la EMR para el tratamiento de lesiones tempranas. Sin embargo, al compararla con la DES, esta última presenta claras ventajas en términos de resecciones en-bloc (96,4\% vs. 50\%), resecciones $\mathrm{R} 0(81,9 \%$ vs. 39,7\%) y riesgo de recurrencia $(2,5 \%$ vs. $25 \%)$ sin grandes diferencias términos de complicaciones ${ }^{23}$. En nuestra opinión, esto sitúa a la DES como la técnica de elección por sobre la EMR.

Grandes estudios de gastrectomía con disección ganglionar han permitido identificar a aquellos pacientes con nulo o muy bajo riesgo de metástasis linfáticas en CGI, hoy conocidos como criterios expandidos ${ }^{24}$, en quienes la DES está validada como una técnica curativa y constituye el estándar de tratamiento. Sin embargo, dichos criterios no aplican para la DES en cáncer esofágico, pues la diseminación linfática es más precoz y la tasa de metástasis linfonodales es $6 \%$ y $20 \%$ para lesiones mucosas y submucosas respectivamente ${ }^{18}$.

Series de esofagectomía han permitido determinar que carcinomas mucosos escamosos y adenocarcinomas, categorizados como M1 (compromete sólo el epitelio) y M2 (invade la lámina propia) presentan nulo o muy bajo riesgo de metástasis linfáticas y, por ende, una resección en bloque R0 sería curati$\mathrm{va}^{25}$. En lesiones M3 (comprometen la muscular de la mucosa) el riesgo es mayor, pero al seleccionar a quienes no presentan permeaciones linfovasculares ronda el 5\% para carcinoma escamoso y es menor al $1 \%$ en adenocarcinoma ${ }^{23,26-28}$. En estos casos debiera preferirse la DES por sobre la cirugía radical, entendiendo que el riesgo de compromiso linfático es menor que la mortalidad asociada a la esofagectomía. Esta afirmación se ve reforzada aún más al comparar sobrevidas globales y específicas por cáncer de esófago a cinco años, para pacientes luego de esofagectomía (80\%-89\% y 94\%-98\%) y luego de DES (92\%-100\% y 96\%-100\%) $)^{27,29-31}$.

En cuanto al compromiso submucoso en cáncer de esófago, los estudios han demostrado que las únicas lesiones susceptibles de tratamiento endoscópico corresponden a adenocarcinomas que comprometen hasta el tercio superficial de la submucosa $(\mathrm{Sm} 1)^{32}$. Para cualquier carcinoma escamoso o adenocarcinoma con profundidad mayor, el riesgo de metástasis linfáticas $\mathrm{y}$, por ende, de un mal resultado oncológico, contraindican la DES como tratamiento definitivo. Consideramos que estas decisiones y la comparación con la esofagectomía, deben ajustarse a la realidad y experiencia local con la cirugía radical y, además, ser individualizadas a cada paciente.

La presente constituye la primera serie reportada de pacientes con cáncer esofágico sometidos a DES en nuestro país. Muestra excelentes resultados oncológicos y seguridad del procedimiento, comparables a las grandes series descritas en la literatura internacional. Sus limitaciones incluyen el pequeño número de pacientes de la serie y el que aún se requiera de un seguimiento a más largo plazo.

Nuestros resultados apoyan la factibilidad y seguridad de la implementación de la DES para el tratamiento de neoplasias esofágicas tempranas en pacientes seleccionados.

\section{Responsabilidades éticas}

Protección de personas y animales. Los autores declaran que para esta investigación no se han realizado experimentos en seres humanos ni en animales.

Confidencialidad de los datos. Los autores declaran que en este artículo no aparecen datos de pacientes.

Conflictos de interés: no hay.

\section{Bibliografía}

1. He Y, Wu Y, Song G, Li Y, Liang D, Jin $\mathrm{J}$, et al. Incidence and mortality rate of esophageal cancer has decreased during past 40 years in Hebei Province, China. Chin J Cancer Res. 2015;27:562 $\square 71$.

2. Bray F, Ferlay J, Soerjomataram I, Siegel RL, Torre LA, Jemal A. Global cancer statistics 2018: GLOBOCAN estimates of incidence and mortality worldwide for 36 cancers in 185 countries. CA Cancer J Clin. 2018;68:394 $\square 424$.

3. Devesa SS, Blot WJ, Fraumeni JF Jr. Changing patterns in the incidence of esophageal and gastric carcinoma in the United States. Cancer 1998;83:2049 $\square 53$. 4. Njei B, McCarty TR, Birk JW. Trends in esophageal cancer survival in United States adults from 1973 to 2009: A SEER database analysis. J Gastroenterol Hepatol. 2016;31:1141ロ6.

5. Low DE, Kuppusamy MK, Alderson D, Cecconello I, Chang A, Darling G. Benchmarking complications associated with esophagectomy. Ann Surg. 2019;269:291 $\square$. 
6. Norero E, Ceroni M, Martínez C, Mejia R, Muñoz R, Godoy C, et al. Complicaciones postoperatorias en esofagectomía por cáncer. Evaluación de 215 casos según definiciones del grupo de consenso internacional. Rev Cirugía 2020;72(5). Disponible en: doi: $10.35687 / \mathrm{s} 2452-$ 45492020005600.

7. Braghetto I, Cardemil H, Korn O, Balladares H, Mandiola C, Yarmuch J, et al. Cirugía mínimamente invasiva en la patología esofágica tumoral: experiencia en 85 pacientes. Rev Chil Cir. 2015;67:218.

8. Csendes A, Braghetto I, Cardemil G, Cortés S, Musleh M. Resultados de la esofaguectomía en 53 pacientes con adenocarcinoma del esófago y Barrett extenso. Rev Chil Cir. 2013;65:121-7.

9. Norero E, Ceroni M, Ramírez A, Mejía R, Martínez C, Muñoz R, et al. Experiencia inicial con esofagectomía mínimamente invasiva transtorácica en posición semiprono por cáncer esofágico. Rev Chil Cir. 2018;70:27-34.

10. Faivre J, Bory R, Moulinier B. Benign tumors of the oesophagus: value of endoscopy. Endoscopy 1978;10:264-8.

11. Guo HM, Zhang XQ, Chen M, Huang SL, Zou XP. Endoscopic submucosal dissection vs endoscopic mucosal resection for superficial esophageal cancer. World J Gastroenterol. 2014;20:5540 $\square$.

12. Park HC, Kim DH, Gong EJ, Na HK, Ahn JY, Lee JH, et al. Ten-year experience of esophageal endoscopic submucosal dissection of superficial esophageal neoplasms in a single center. Korean $\mathrm{J}$ Intern Med. 2016;31:1064 $\square 72$.

13. Purchiaroni F, Costamagna G, Hassan C. Quality in upper gastrointestinal endoscopic submucosal dissection. Ann Transl Med. 2018;6:261.

14. Abdelfatah MM, Barakat M, Ahmad D, Ibrahim M, Ahmed Y, Kurdi Y, et al. Longterm outcomes of endoscopic submucosal dissection versus surgery in early gastric cancer: a systematic review and metaanalysis. Eur J Gastroenterol Hepatol.
2019;31:418-24.

15. Jeon HK, Kim GH, Lee BE, Park DY, Song GA, Kim DH, et al. Long-term outcome of endoscopic submucosal dissection is comparable to that of surgery for early gastric cancer: a propensitymatched analysis. Gastric Cancer 2018; 21:133-43.

16. Cho JW, Choi SC, Jang JY, Shin SK, Choi $\mathrm{KD}$, Lee JH, et al. Lymph node metastases in esophageal carcinoma: an endoscopists view. Clin Endosc. 2014;47:523 $\square$ 9.

17. Sundelöf M, Lagergren J, Ye W. Patient demographics and lifestyle factors influencing long-term survival of oesophageal cancer and gastric cardia cancer in a nationwide study in Sweden. Eur J Cancer 2008;44:1566 71 .

18. Pennathur A, Farkas A, Krasinskas AM, Ferson P, Goodinf W, Gibson M, et al. Esophagectomy for T1 esophageal cancer: outcomes in 100 patients and implications for endoscopic therapy. Ann Thorac Surg. 2009;87:1048 $\square 55$.

19. Inoue $H$, Endo $M$, Takeshita $K$, Yoshino K, Muraoka Y, Yoneshima H. Endoscopic esophageal mucosal resection using a cap-fitted panendoscope (EMRC). Surg Endosc. 1992;6:264-5.

20. Makuuchi H, Yoshida T, Ell C. Four step endoscopic esophageal mucosal resection (EEMR) tube method of resection for early esophageal cancer. Endoscopy 2004;36:1013-8.

21. Momma K, Yoshida M, Yamada Y. Endoscopic mucosectomy of early esophageal cancer. Stomach Intest. 1993;28:141-51.

22. Oyama T, Tomori A, Hotta K, Morita S, Kominato K, Tanaka M, et al. Endoscopic submucosal dissection of early esophageal cancer. Clin Gastroenterol Hepatol. 2005;3:S67-70.

23. Ishihara $\mathrm{R}$, Arima $\mathrm{M}$, Iizuka $\mathrm{T}$, Oyama T, Katada C, Kato M, et al. Endoscopic submucosal dissection/endoscopic mucosal resection guidelines for esophageal cancer. Digestive Endoscopy 2020;32:452-93.

24. Gotoda T, Yanagisawa A, Sasako M,
Ono H, Nakanishi Y, Shimoda T, et al. Incidence of lymph node metastasis from early gastric cancer: estimation with a large number of cases at two large centers. Gastric Cancer 2000;3:219-25.

25. Kitagawa Y, Uno T, Oyama T, Kato K, Kato H, Kawahubo H, et al. Esophageal cancer practice guidelines 2017 edited by the Japan esophageal society. Esophagus 2019;16:1-43.

26. Ishihara $\mathrm{R}$, Oyama $\mathrm{T}$, Abe $\mathrm{S}$, Takahashi $\mathrm{H}$, Ono H, Fujisaki J, et al. Risk of metastasis in adenocarcinoma of the esophagus: A multicenter retrospective study in a Japanese population. J Gastroenterol. 2017;52:800-8.

27. Lorenz D, Origer J, Pauthner M, Graupe F, Fisseler-Eckhoff A, Stolte M, et al. Prognostic risk factors of early esophageal adenocarcinomas. Ann Surg. 2014;259:469-76.

28. Aida J, Ishizaki T, Arai T, Takubo K. Prognostication of superficial Barrett's carcinoma: A Japanese multicenter study. Hum Pathol. 2018;76:156-66.

29. Probst A, Aust D, Markl B, Anthuber M, Messmann H. Early esophageal cancer in Europe: Endoscopic treatment by endoscopic submucosal dissection. Endoscopy 2014;47:113-21.

30. Dubecz A, Kern M, Solymosi N, Schweigert M, Stein H. Predictors of lymph node metastasis in surgically resected T1 esophageal cancer. Ann Thorac Surg. 2015;99:1879-85.

31. Abe S, Ishihara R, Takahashi H, Ono H, Fujisaki J, Matsui A, et al. Long-term outcomes of endoscopic resection and metachronous cancer after endoscopic resection for adenocarcinoma of the esophagogastric junction in Japan. Gastrointest Endosc. 2019;89:1120-8.

32. Manner H, Pech O, Heldmann Y, May A, Pohl J, Behrens A, et al. Efficacy, safety, and long-term results of endoscopic treatment for early stage adenocarcinoma of the esophagus with low-risk sm1 invasion. Clin Gastroenterol Hepatol. 2013;11:630 $\square$ e45. 